\title{
MULTIDIMENSIONAL CHARACTERISATION OF TIME-DEPENDENT IMAGE DATA: A CASE STUDY FOR THE PERIPHERAL NERVOUS SYSTEM IN AGEING MICE
}

\author{
Matthias WeBer ${ }^{\bowtie}$, ThOMAs Wilhelm AND Volker SchmidT \\ Institute of Stochastics, Ulm University, 89069 Ulm, Germany \\ e-mail: matthias.weber@uni-ulm.de, thomas.wilhelm@uni-ulm.de, volker.schmidt@uni-ulm.de \\ (Received January 2, 2021; revised May 4, 2021; accepted May 17, 2021)
}

\begin{abstract}
Segmentation of $\mu \mathrm{m}$-resolution image data of irregularly shaped objects poses challenges to existing segmentation algorithms. This is especially true, when imperfections like noise, uneven lightning or traces of sample preparation are present in the image data. In this paper, considering electron micrographs of femoral quadriceps nerve sections of mice, a segmentation method to extract single axons surrounded by myelin sheaths is developed which is able to cope with various imperfections and artefacts. This approach successfully combines established methods like local thresholding and marker-based watershed transform to achieve a reliable segmentation of the given data. Indeed, the resulting segmentation map can be used to quantitatively determine geometrical characteristics of the axons and myelin sheaths. This is exemplified by modelling the joint probability distribution of axon area and myelin sphericity using a parametric copula approach, and by analysing the evolution of the model parameters for image data obtained from mice of different ages.
\end{abstract}

Keywords: automated segmentation, copula, electron microscopy, parametric modelling, quadriceps nerve, statistical image analysis.

\section{INTRODUCTION}

Automated segmentation of images of granular structures often poses challenges specific to the structures in question. Measuring structures necessarily introduces imperfections and artefacts like noise or potentially poor contrast, and a discretised image often suffers from inadequate resolution. While many of these issues can be overcome by improving imaging techniques, the improvements are often a trade-off between resolution and measured area or time steps, and signal-to-noise ratio. Dealing with the available techniques and image data thus requires involved algorithms to perform image segmentation and extract the desired information. This is often due to the fact that resolution and noise do not allow for detecting gaps between single objects and thus lead to larger connected regions consisting of many objects. While these regions can often be identified by (local) thresholding or clustering techniques like k-means clustering, a segmentation into single objects requires further efforts. Many available techniques for singleobject segmentation make implicit assumptions about the structures of interest. For example, the widelyused watershed algorithm (Beucher and Lantuéjoul, 1979; Roerdink and Meijster, 2000) theoretically requires convex objects to perform well without further improvements. As a result, the watershed approach often suffers from over-segmentation. The marker- based watershed addresses this issue by explicitly choosing the approximate objects' centroids. Other algorithms like model-based segmentation even more explicitly assume specific shapes, e.g., spherical objects. Moreover, many algorithms assume objects without holes or cracks. These limitations can be overcome by, e.g., filling holes and cracks prior to segmentation and later re-introducing them into the segmented objects. However, this approach still relies on quite regularly shaped objects with only small holes and cracks. These assumptions are not valid for image data of ring-like objects with non-spherical shape on which we focus in the present paper. For instance, electron micrographs of femoral quadriceps nerve sections of mice show a bundle of axons, each surrounded by a myelin sheath. In recent years, novel approaches to image segmentation based on convolutional neural networks have been developed. For the present type of data, e.g., AxonDeepSeg (Zaimi et al., 2018) is a recent open-source tool for image segmentation. However, this tool performs only a phase-based segmentation and no single-object segmentation. Moreover, the accuracy varies hugely depending on the size of objects. These limitations make the development of a reliable tool for objectwise segmentation necessary.

In the present paper, we propose a segmentation method suitable for image data of ring-like objects with non-spherical shape. The shapes are similar to 
the (dilated) boundary of clover leafs or stars. We apply our technique to electron micrographs of femoral quadriceps nerve sections of mice, where the nerve is composed of a bundle of axons, each surrounded by a myelin sheath. The sheaths form ring-like objects and often touch neighbouring sheaths such that a naive segmentation is not feasible. Moreover, the image data exhibits some imperfections resulting in locally lost information, see Fig. 1. We therefore develop a multistep segmentation method consisting of a sequence of preprocessing steps leading to a binary image where the myelin sheaths are separated from background and axons, followed by a subsequent segmentation of this binary image into single myelin sheaths. The segmentation algorithm is capable of dealing with the intricate shapes of myelin sheaths and their hollow interior. For this, we automatically process the binary image into a carefully designed set of markers and a gradient map for the watershed algorithm. The choice of markers ensures that no over-segmentation occurs whereas the gradient map is chosen such that the watershed lines split neighbouring myelin sheaths at the proper locations.

(b)
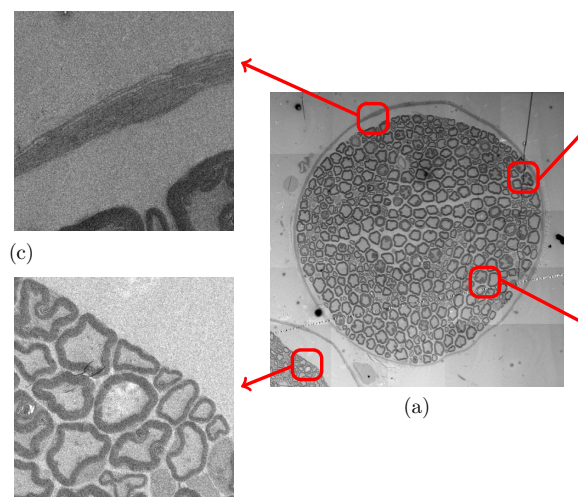

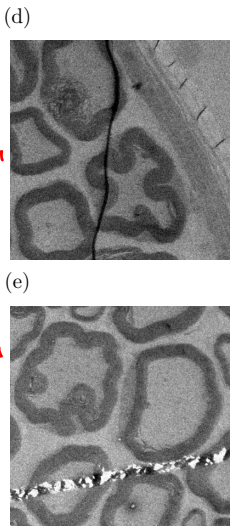

Fig. 1. Original greyscale image showing a 6 months old nerve (a). Different imperfections which are removed by the multi-step segmentation method developed in this paper, like the perineurium (b), other visible nerves (c), dark wrinkles $(d)$, and further traces of sample preparation (e).

The used electron micrographs show nerve sections from 6, 12 and 24 months old mice. During ageing, natural effects change the structure of axons and myelin sheaths which results in changes of their sizes and shapes. The segmentation of the data into single axons surrounded by myelin sheaths enables us to geometrically characterise each pair of axon and myelin sheath and statistically analyse their properties and the effects of ageing. We exemplify this by modelling the joint probability distribution of axon area and myelin sphericity using a parametric copula approach, and by analysing the evolution of the model parameters for image data obtained from mice of different ages.

The rest of this paper is organised as follows. First, we explain the methods which have been used for acquisition and preprocessing of image data, including binarisation and subsequent singleobject segmentation. Then, the results of statistical analysis and parametric modelling of uni- and bivariate probability distributions of axon area and myelin sphericity for 6, 12 and 24 months old mice is explained. Furthermore, a method of parameter interpolation is proposed to predict the distribution of geometrical nerve descriptors for mice ages where no experimental image data is available. Finally, we discuss the obtained results.

\section{MATERIALS AND METHODS}

In this section, we explain the methods which have been used for sample preparation and image preprocessing. Dividing the images of nerves into single axons and their surrounding myelin sheaths requires an object-based segmentation. In preparation for the actual segmentation of the images, a partly sample-specific preprocessing is performed which adjusts for local deviations in illumination and removes some further imperfections.

\section{SAMPLE PREPARATION AND IMAGING}

Sample preparation was performed as reported in Yuan et al. (2018). For electron microscopy, sacrificed mice were transcardially perfused with $4 \%$ PFA and 2\% glutaraldehyde in $0.1 \mathrm{M}$ cacodylate buffer. Dissected femoral nerves were subsequently postfixed in the same solution overnight at $4^{\circ} \mathrm{C}$, followed by osmification ( $2 \%$ OsO4), dehydration, and embedding in Spurr's medium. Ultrathin cross sections $(80 \mathrm{~nm})$ of femoral nerves were mounted onto copper grids and counterstained with lead citrate. Electron micrographs were taken with a Slow Scan charge-coupled device, short CCD, (ProScan) camera mounted to a Leo $906 \mathrm{E}$ transmission electron microscope (Zeiss) and corresponding software iTEM (soft imaging system). Multiple image alignments (MIA) were acquired at a primary magnification of 400x for covering cross-sections of whole femoral quadriceps nerve sections.

\section{IMAGE PREPROCESSING}

Due to the fact that images are taken by means of electron microscopy, some illumination effects are present in the greyscale images. For example, there are 
dark artefacts like wrinkles of the nerves which arise during mounting the samples onto the copper grids, partly visible other nerves and the perineurium, as well as other traces of sample preparation. These undesired objects can be seen in the small cutouts of Fig. 1 (b), (c), (d) and (e). They are making a simple computerbased detection of single axons and myelin sheaths difficult.

For this reason, we are first preprocessing the images with morphological and histogrambased methods which are described in detail, e.g., in Heumans et al. (1992); Soille (2013); Vincent (1989). A histogram-based classification is employed to remove artefacts like wrinkels and uneven illumination, because they make it difficult to distinguish clearly between different single axons.

\section{Removement of Wrinkels and Other Sample Preparation Traces}

Artefacts consisting of dark pixels are detected using thresholding. The result of this step is a binary image, see Fig. 2 (b), where the detected pixels are now white. This binary image is used as a mask for a Gaussian filter, see, e.g., Weber et al. (2020) where this kind of preprocessing procedure has been applied to cryo-EM images of murine amyloid protein fibrils. The Gaussian filter serves to eliminate the detected artefacts. In Fig. 2 (d) and (e) the same cutouts as in Fig. 1 (d) and (e) are shown, where the artefacts (i.e. wrinkles in Fig. 2 (d) and other traces of sample preparation in Fig. 2 (e)) are now deleted and single axons can be separated later on, see Section 3.2 below. Note however that some artefacts like wrinkles, see Fig. 1 (d) and 2 (d), are not completely deleted. In particular, wrinkles lying on top of dark myelin sheaths are not removed, but this does not affect the segmentation performed later on. It is important that myelin sheaths are not wrongly connected by artefacts anymore. The fat blue arrows in Fig. 2 (d) and (e) are pointing at separated myelin sheaths after applying the Gaussian filter.

In a final step, we visually inspect the preprocessed greyscale images and manually correct remaining artefacts which may lead to imperfections in the final segmentation. This is only necessary for a small fraction of the image and is done by manually labelling some pixels as myelin or background.

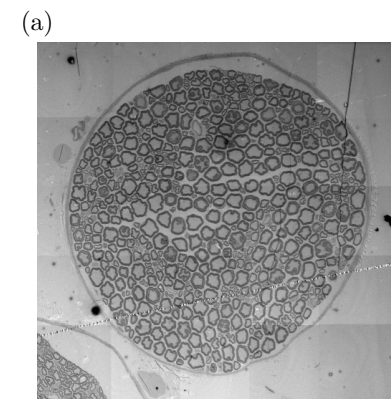

(c)

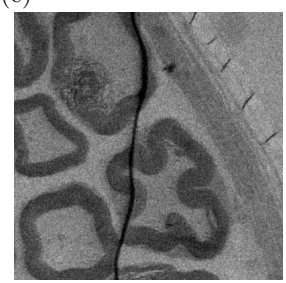

(e)

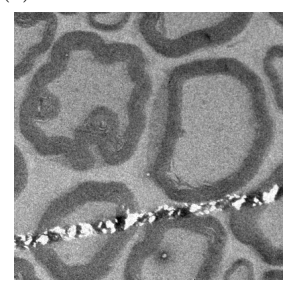

(b)

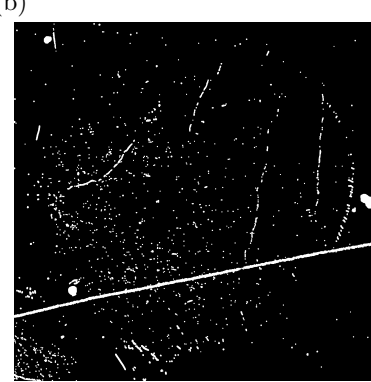

(d)

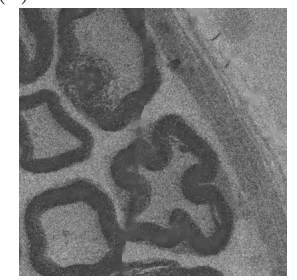

(f)

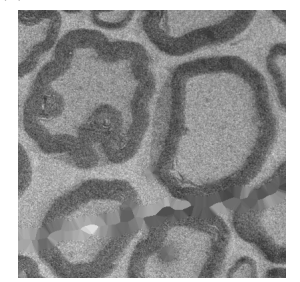

Fig. 2. Detecting artefacts in the original greyscale image (a) by thresholding $(b)$. The result, $(d)$ and $(f)$, after re-calculating the pixel values in the affected regions, $(c)$ and (e), see also Fig. $I(d)$ and $(e)$.

\section{Manual Extraction of the Nerve}

Subsequently a mask is created to extract the nerve. In this way, partly visible other nerves and the sheath around the whole nerve, the so-called perineurium, are excluded from the segmentation.

\section{SEGMENTATION}

The previous preprocessing steps yield a greyscale image of the nerve which has been improved with respect to different artefacts and imperfections. However, the actual segmentation of this image still poses a challenging problem as the underlying objects are highly non-spherical and hollow. As contrast and greyscale levels vary across the preprocessed image, a local thresholding serves to simplify the data without losing information. The binarised image is then segmented into single objects using a specially tailored watershed approach.

\section{BINARISATION}

Compared to a local environment, the myelin sheaths are darker than the surrounding image. 
While uneven illumination makes it impossible to use a global threshold to separate myelin from the background, a local thresholding approach (Gonzalez and Woods, 2007) is feasible. Here, the value of each pixel is compared to the weighted average value in a defined region around the pixel. For weights, we choose a Gaussian kernel with standard deviation of 25 px and a cut-off distance of $80 \mathrm{px}$. A pixel is classified as myelin if its value is less than the weighted mean value of the surrounding pixels. The phase-related segmentation result of a cutout of the 6 months old nerve is shown in Fig. 3 (a).

\section{SINGLE-OBJECT SEGMENTATION}

The binarised image contains sufficient information about all myelin sheaths to identify single myelinated axons. This single-object segmentation is performed using a marker-based watershed transform (Lantuéjoul, 1982; Roerdink and Meijster, 2000). For this, we need a set of markers within the myelinated axons and a gradient image for the watershed to perform on.

For both, we first extract the axons present in the binarised image, see Fig. 3 (b). Note that each axon is a separate connected component in the black phase of the binary image, see Fig. 3 (a). Furthermore, we assume that the background consists of a single connected component which can be easily identified by size. Thus, by computing the area of the connected components (Hoshen and Kopelman, 1976) of the black phase and removing the background, we obtain a family of more or less circular regions representing the axons, see Figs. 3 and 4.

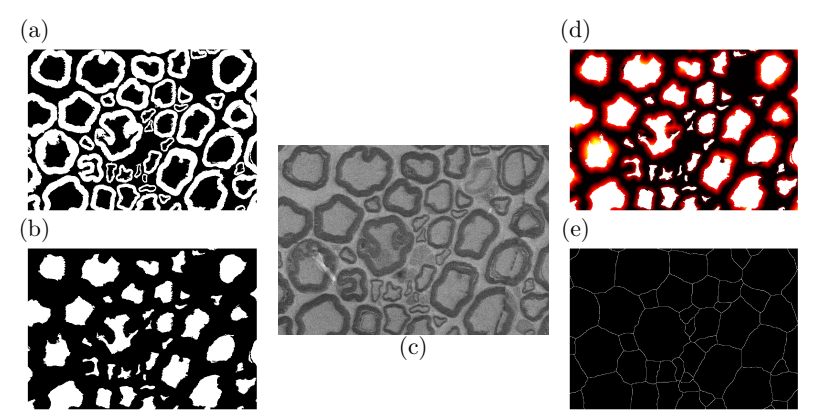

Fig. 3. Cutouts showing the myelin sheaths (a), the detected axons $(b)$ and the corresponding part of the original image (c). Modified distance transform with respect to the background, with watershed markers corresponding to the axons shown in white $(d)$ and resulting watershed lines $(e)$.
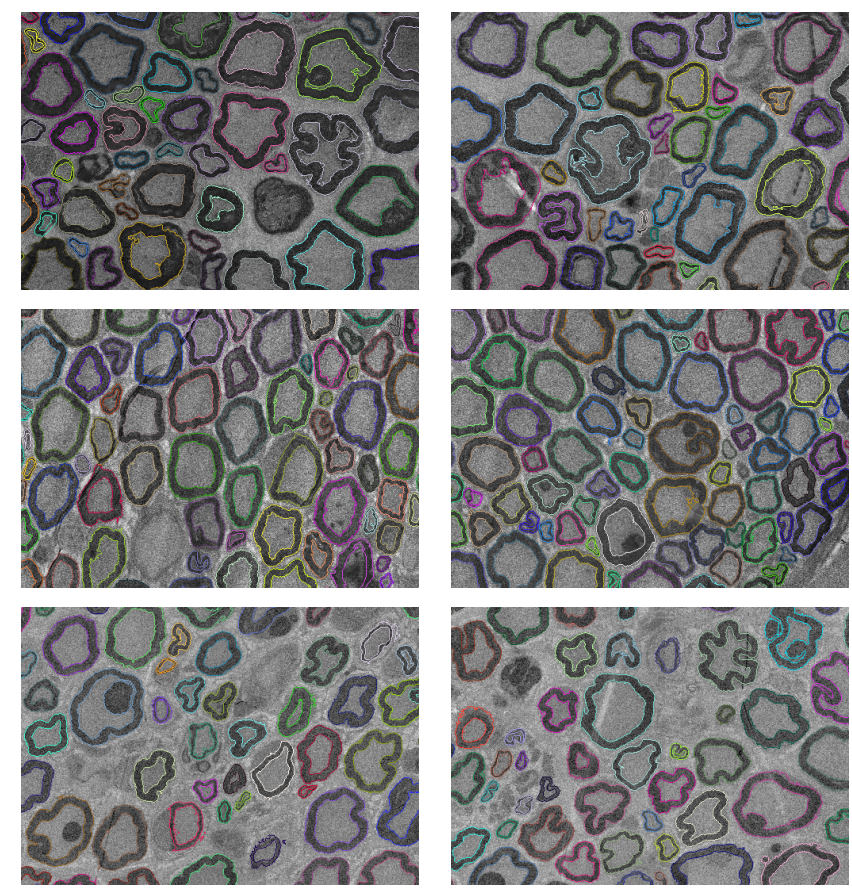

Fig. 4. Cutouts showing 6, 12 and 24 months old nerves (from top to bottom). Extracted myelin sheaths are outlined in different colors. The axons correspond to the areas within the inner boundaries of the myelin sheaths.

We chose the whole axons as markers for the marker-based watershed, see Fig. 3 (d). This ensures that the axons are never split into separate regions by the watershed transform. Furthermore, we compute the distance transform with the myelinated axons with respect to the background. We use the inverse of this distance transform as gradient image for the markerbased watershed to perform on. This ensures that no watershed lines will be placed inside an axon and, on the other hand, watershed lines are placed inside the background region whenever this is possible, see Fig. 3 (e). The final result, i.e., the myelin sheaths extracted in this way from the binarised image, is visualised in Fig. 4. These cutouts show the accuracy of the proposed method. While the shape of most myelin sheaths is accurately detected and the watershed segmentation separates neighbouring myelin sheaths well, some artefacts would pose problems to the local thresholding and were therefore removed by hand.

By visual inspection of the extracted axons and myelin sheaths shown in Fig. 4 we can hardly see the changes in the structure of axons and myelin sheaths during ageing. Therefore, a deeper statistical analysis of the segmented image data is necessary. This will be done below, together with parametric modelling of uniand bivariate probability distributions of size and shape descriptors of axons and myelin sheaths. In this way, age-dependent changes of the structure of axons and myelin sheaths can be revealed and quantified. 


\section{RESULTS}

Application of the segmentation techniques described in the previous section yields a set of axons and corresponding myelin sheaths for each image, where 1598, 1565 and 1996 axons and myelin sheaths have been extracted from planar nerve sections of 6,12 and 24 months old mice, respectively. Altogether, size and shape information of more than 5100 axons and myelin sheaths is available. Thus, for each pair of axons and myelin sheaths, we can compute geometrical descriptors relating to functional properties and statistically analyse the resulting data. Moreover, by fitting parametric distributions, we can characterise age-dependent changes of the structure of mice nerves.

In comparison to a purely histogram-based evaluation of geometrical nerve descriptors, the fitting of parametric distributions allows for a more comprehensive quantification of ageing effects. While aggregated quantities, like mean and standard deviation, computed from raw histograms provide only partial information about the underlying distributions, the parameters and, in particular, the temporal changes of parameters of the fitted distributions fully characterise their evolution in time. For example, in general, probabilities for exceeding an arbitrary specified threshold can not be reconstructed from aggregated quantities like mean and standard variation, but can be obtained from the knowledge of the parametric model type along with the estimated parameters.

\section{ANALYSIS OF AXON AREA FOR DIFFERENT AGES}

For each axon extracted from the available greyscale images, we compute its area, denoted by $A$ in the following, by counting the corresponding number of pixels. Fig. 5 shows the histograms of axon area for 6, 12 and 24 months old nerves. Mean values and standard deviations are given in Table 1. Looking at the mean values, we see that there is an increase of mean axon area in the time interval between 6-12 months, and a decrease between 12-24 months.
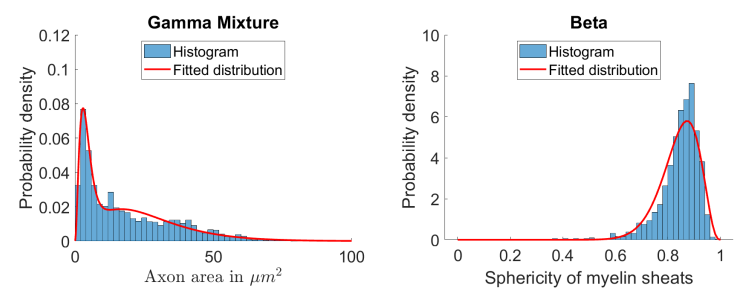
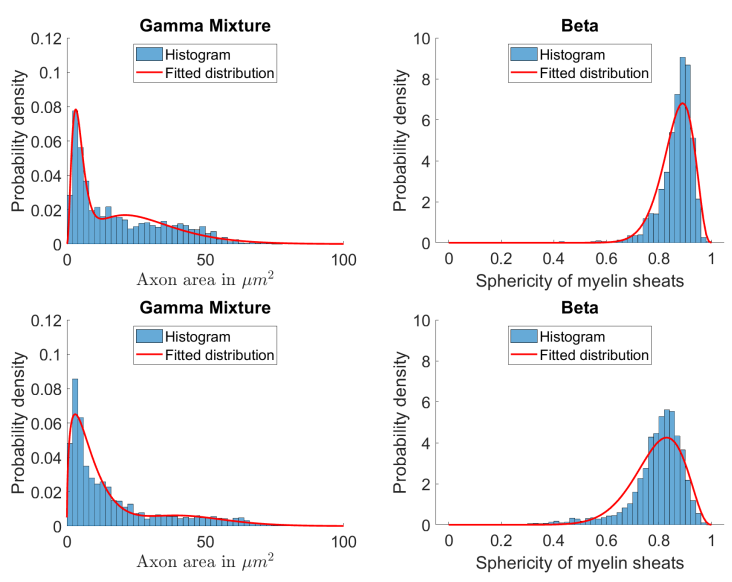

Fig. 5. Histograms and fitted distributions of axon area and myelin sphericity for 6,12 and 24 months old nerves (from top to bottom). Note that the values of the histograms are normalized.

\begin{tabular}{|l||c|c|c|}
\hline \multicolumn{4}{|c||}{ age Axon area } \\
\begin{tabular}{|l||c|c|c|}
\hline mean & 18.44 & 19.03 & 16.51 \\
sd & 16.64 & 16.83 & 17.73 \\
\hline \hline mean (small axons) & 3.68 & 3.76 & 3.53 \\
sd (small axons) & 1.64 & 1.63 & 1.67 \\
\hline mean (medium axons) & 16.23 & 16.18 & 14.97 \\
sd (medium axons) & 5.89 & 5.98 & 5.59 \\
\hline mean (large axons) & 42.48 & 42.26 & 48.06 \\
sd (large axons) & 10.94 & 9.49 & 12.62 \\
\hline
\end{tabular}
\end{tabular}

Myelin sphericity
\begin{tabular}{|l||c|c|c|}
\hline mean & 0.84 & 0.87 & 0.79 \\
sd & 0.08 & 0.07 & 0.10 \\
\hline \hline mean (small axons) & 0.83 & 0.86 & 0.79 \\
sd (small axons) & 0.09 & 0.08 & 0.11 \\
\hline mean (medium axons) & 0.85 & 0.87 & 0.79 \\
sd (medium axons) & 0.07 & 0.06 & 0.10 \\
\hline mean (large axons) & 0.85 & 0.87 & 0.82 \\
sd (large axons) & 0.06 & 0.06 & 0.08 \\
\hline
\end{tabular}

\begin{tabular}{|l||l|l|l|}
\multicolumn{4}{|c|}{ Size of subsamples } \\
\hline small axons & 587 & 583 & 868 \\
medium axons & 596 & 533 & 735 \\
large axons & 415 & 449 & 393 \\
\hline
\end{tabular}

Table 1. Mean value and standard deviation of axon area (in $\mu^{2}$ ) and myelin sphericity for total population and for axons with area-equivalent diameter smaller than $3 \mu \mathrm{m}$, between 3-6 $\mu \mathrm{m}$, and larger than $6 \mu \mathrm{m}$ of 6,12 and 24 months old nerves.

To better understand possible reasons for the change of mean axon area between 6, 12 and 24 months, we subdivided the data into axons with small, 
medium and large area-equivalent diameter (i.e., the diameter of a circle with equal area), where small means that the area-equivalent diameter is smaller than $3 \mu \mathrm{m}$, medium that it is between 3-6 $\mu \mathrm{m}$, and large that it is larger than $6 \mu \mathrm{m}$. When looking at Table 1 , we see that there is a slight decrease of mean area for small and medium axons between 12 and 24 months, whereas the mean area of large axons drastically increases. At first glance, the latter effect seems to be contradictory to the decrease of global mean axon area between 12 and 24 months stated in Table 1 . However, when looking at the sizes of subsamples for 24 months old mice, it becomes clear that the subsample sizes drastically increase for small and medium axon and, simultaneously, slightly decrease for large axons, due to a left shift of the distribution of medium and large axon areas in the time interval between 12 and 24 months.

Furthermore, globally, the standard deviation of axon areas slightly increases over time, see Table 1 . The main reason for this is the considerable increase of standard deviation of large axon areas in the time interval between 12 and 24 months (from 9.49 to 12.62).

An efficient way to characterise age-dependent changes of structural properties of mice nerves is to use parametric distribution models. Then, for each age, we obtain a set of parameters characterising the whole distribution of a geometrical descriptor of nerves, e.g., of axon area. Note that several types of parametric distributions may be fitted to the given data. For an overview of various families of univariate probability distributions, see Bickel and Doksum (2015). To parametrically model the distribution of axon areas, we used a mixture of two gamma distributions whose probability density is given by

$$
\begin{aligned}
f_{\omega, \alpha_{1}, \alpha_{2}, \beta_{1}, \beta_{2}}(x)= & \omega \frac{1}{\beta_{1}^{\alpha_{1}} \Gamma\left(\alpha_{1}\right)} x^{\alpha_{1}-1} e^{\frac{-x}{\beta_{1}}}+ \\
& (1-\omega) \frac{1}{\beta_{2}^{\alpha_{2}} \Gamma\left(\alpha_{2}\right)} x^{\alpha_{2}-1} e^{\frac{-x}{\beta_{2}}}
\end{aligned}
$$

for parameters $\alpha_{1}, \alpha_{2}, \beta_{1}, \beta_{2}, x>0, \omega \in[0,1]$.

Fig. 5 shows the histograms of axon area along with the fitted mixed gamma distributions, where the estimated parameter values are given in Table 2. It turns out that all model parameters exhibit interesting differences of trend in the time intervals between 6-12 months and 12-24 months, respectively, see also Fig. 7.

\begin{tabular}{|c||c|c|c|c|c|}
\hline age & $\alpha_{1}$ & $\beta_{1}$ & $\alpha_{2}$ & $\beta_{2}$ & $\omega$ \\
\hline 6 & 2.78 & 9.70 & 3.17 & 1.13 & 0.63 \\
\hline 12 & 3.50 & 8.42 & 3.26 & 1.38 & 0.58 \\
\hline 24 & 9.98 & 4.65 & 1.50 & 5.92 & 0.20 \\
\hline
\end{tabular}

\begin{tabular}{|c||c|c||c|}
\hline age & $a$ & $b$ & $\theta$ \\
\hline 6 & 20.92 & 3.90 & 0.0807 \\
\hline 12 & 26.00 & 4.08 & 0.0754 \\
\hline 24 & 13.56 & 3.58 & 0.0978 \\
\hline
\end{tabular}

Table 2. Estimated parameter values of the fitted mixed gamma distributions for axon area $\left(\alpha_{1}, \alpha_{2}, \beta_{1}, \beta_{2}, \omega\right)$, the fitted beta distributions for myelin sphericity $(a, b)$ and the copula describing the joint distribution $(\theta)$ of 6, 12 and 24 months old nerves.

\section{ANALYSIS OF MYELIN SPHERICITY FOR DIFFERENT AGES}

As a second descriptor, complementary to axon area, we consider the so-called sphericity of the myelin sheaths. Note that the sphericity of a planar geometrical object extracted from image data is given by the ratio

$$
S=\frac{C_{e}}{C}
$$

of the circumference $C_{e}$ of the area-equivalent circle and the circumference $C$ of the object itself. As a ratio of two lengths, the quantity $S$ defined above is dimensionless. The sphericity of an object depends on its shape and, in particular, on its roundness and takes values between 0 and 1. For a higher value of $S$ the shape of the considered object is closer to the shape of a circle. Note that geology (Wadell, 1932) was one of the first fields where shape analysis of non-spherical objects was performed, see also (Furat et al., 2019b) for more recent applications to objects with complex (non-spherical) shapes extracted from image data.

For the given data of mice nerves, it turns out that there is a considerable change in the distribution of sphericity between 12 and 24 months. On the other hand, over the first 12 months the distribution of the sphericity seems to change less, see Fig. 5. Thus, the sphericity of myelin sheaths behaves similarly in time in comparison to the axon area considered earlier.

Moreover, when separately considering axons with area-equivalent diameters smaller than $3 \mu \mathrm{m}$, between 3-6 $\mu m$, and larger than $6 \mu m$, respectively, it turns out that the mean values of myelin sphericity of these subsamples behave similar as the mean value of myelin sphericity of the entire sample at the corresponding time points, see Table 1.

However, the changes in the distribution of sphericity observed in Fig. 5 are much better reflected by the probability $\mathbb{P}(S<s)$ that the myelin sphericity $S$ is smaller than a certain predefined threshold $s \in[0,1]$, see Table 3. For example, for $s=0.85$ this probability clearly decreases in the time interval between 6 and 12 months and increases between 12 and 24 months. 


\begin{tabular}{|c||c|c|c|}
\hline age & 6 & 12 & 24 \\
\hline $\mathbb{P}(S<0.85)$ (total) & 0.437 & 0.287 & 0.703 \\
\hline \hline $\mathbb{P}(S<0.85)$ (small) & 0.491 & 0.360 & 0.702 \\
\hline $\mathbb{P}(S<0.85)$ (medium) & 0.379 & 0.275 & 0.758 \\
\hline $\mathbb{P}(S<0.85)$ (large) & 0.446 & 0.205 & 0.603 \\
\hline
\end{tabular}

Table 3. Level crossing probabilities of myelin sphericity for the entire sample of all axons, for axons with area-equivalent diameter smaller than $3 \mu \mathrm{m}$, between 3-6 $\mu \mathrm{m}$, and larger than $6 \mu \mathrm{m}$ of 6,12 and 24 months old nerves

Similar to the modelling of axon area by parametric distributions, we considered gamma, lognormal, Weibull and beta distributions for modelling myelin sphericity and estimated the corresponding parameters. The globally best fit was obtained using beta distributions, which are twoparametric distributions given by the density

$$
f_{a, b}(y)=\frac{1}{B(a, b)} y^{a-1}(1-y)^{b-1}, \quad a, b>0, y \in[0,1] .
$$

Fig. 5 shows the histograms of myelin sphericity along with the fitted beta distributions, where the estimated parameter values are given in Table 2. Again, both model parameters, $a$ and $b$, exhibit interesting inversions of trend in the time intervals between 612 months and 12-24 months, respectively, where both parameters first increase and then decrease, see also Fig. 7.

\section{BIVARIATE DISTRIBUTION MODELS}

To look just at univariate histograms of single geometrical nerve descriptors can be problematic, because there is no information included regarding the correlation between the different descriptors. It is well known (Friede, 1986; Smith et al., 1982) that the geometries of myelin sheaths and axons are related with each other. A computation of Pearson's linear correlation coefficient (Bickel and Doksum, 2015), denoted by $\rho$, of the area of axons and the sphericity of myelin sheaths for 6, 12 and 24 months old nerves confirms this, see Table 4.

\begin{tabular}{|c||c|c|c|}
\hline age & 6 & 12 & 24 \\
\hline$\rho$ & 0.03 & 0.11 & 0.14 \\
\hline
\end{tabular}

Table 4. Pearson's linear correlation coefficient $\rho$ of the area of axons and the sphericity of corresponding myelin sheaths.

Therefore, it is necessary to take correlation effects into account when modelling the bivariate probability distribution of axon area and myelin sphericity. Again, similar to the situation considered previously, the fitting of a two-dimensional parametric distribution model allows for a more comprehensive quantification of ageing effects compared to the exclusive computation of aggregated statistical descriptors like mean values and covariances from the two-dimensional histograms.

In the literature dealing with statistical analysis of image data, Gaussian distributions are often used because of their simple closed form. In particular, it is easily possible to fit a multidimensional Gaussian model to available data, using the maximum likelihood method. However, looking at the distributions of axon area and myelin sphericity visualised in Fig. 5, it becomes obvious that they are clearly not Gaussian at any time point. Moreover, because of the correlation between these characteristics shown in Table 4, the standard method of a bivariate product distribution is not working either. Therefore, we use a copula approach (Durante and Sempi, 2010; Joe, 1997) for modelling the joint distribution of axon area and myelin sphericity. Note that the copula approach has demonstrated its benefits in various other applications in order to fit parametric models to multivariate probability distributions, see, e.g., Furat et al. (2019a); Neumann et al. (2021); von Loeper et al. (2020). A short introduction to this topic is given in the following section. More details about copulas can be found, e.g., in Durante and Sempi (2010) and, in particular, how to estimate their parameters in Joe (1997).

\section{Fitting a Parametric Copula}

Copulas provide a useful framework for parametrically modelling multivariate probability distributions. They represent the correlation structure within the multivariate distribution. In the twodimensional case, where $A$ is a (one-dimensional) random variable with cumulative distribution function $F_{A}$ and $S$ is a (one-dimensional) random variable with cumulative distribution function $F_{S}$, the joint cumulative distribution function $F_{A, S}$ of the (twodimensional) random vector $(A, S)$ with $F_{A, S}(x, y)=$ $P(A \leq x, S \leq y)$ can be represented by

$$
F_{A, S}(x, y)=C\left(F_{A}(x), F_{S}(y)\right), \quad-\infty<x, y<\infty,
$$

where $C$ is a so-called copula, i.e., a suitably chosen two-dimensional cumulative distribution function with marginals being uniform distributions on $[0,1]$. This representation of the joint cumulative distribution function $F_{A, S}$ is ensured by Sklar's theorem (Sklar, 1959). Various classes of parametric copulas can be used to model $C$ which, together with $F_{A}$ and $F_{S}$, characterises the bivariate probability distribution. 
In the following, we model the joint distribution of axon area and myelin sphericity by estimating the parameter $\theta>0$ of a so-called Clayton copula, which is given by

$$
C_{\theta}(u, v)=\left(u^{-\theta}+v^{-\theta}-1\right)^{-\frac{1}{\theta}}, \quad x, y \in[0,1] .
$$

Note that we choose the Clayton copula from the class of Archimedean copulas in favour of AliMikhail-Haq, Frank, Gumbel and Joe copulas (Joe, 1997) as the best fit to the empirical bivariate distribution of axon area and myelin sphericity is obtained using a Clayton copula. Recall that the univariate cumulative distributions functions $F_{A}$ and $F_{S}$ are obtained from the parametric models fitted earlier. Then, the parameter $\theta$ is estimated using the maximum likelihood method. The estimated values of $\theta$ are given in Table 2.

The fitted Clayton copula, together with the (mixed) gamma and beta distributions for axon area and myelin sphericity, provides a parametric characterisation of the (joint) bivariate probability distribution of axon area and myelin sphericity, see Fig. 8.

\section{Computation of Conditional Distributions}

The representation of the joint bivariate distribution of axon area $A$ and myelin sphericity $S$ via Clayton copulas, visualised in Fig. 8, facilitates further analyses of the age-dependent morphology of mice nerves. In particular, using Equation (1) the conditional probability $P\left(S<s \mid a_{1}<A<a_{2}\right)$ can be computed that the sphericity of myelin sheaths takes values smaller than some threshold $s$, under the condition that their axon areas belong to a given range of values, e.g. to the interval $\left[a_{1}, a_{2}\right]$ for some $a_{1}, a_{2}>0$.

Fig. 6 shows conditional distributions of myelin sphericity for the different ages. We can clearly see that small axons tend to have less rounded myelin sheaths than medium and large axons. This effect is already visible in young nerves, but gets stronger for later ages and can thus be interpreted as another structural effect of ageing.
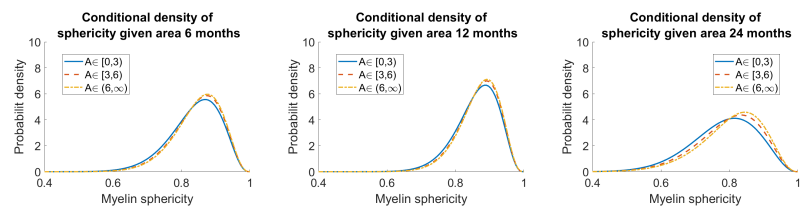

Fig. 6. Conditional distributions of myelin sphericity $S$ for given ranges of axon area $A$ and three different ages. The three different ranges of axon area correspond to area-equivalent diameters $<3 \mu \mathrm{m}, 3-$ $6 \mu \mathrm{m}$ and $>6 \mu \mathrm{m}$, respectively.

\section{PREDICTIVE MODELS BY PARAMETER INTERPOLATION}

Another important benefit of parametric distribution models is the possibility to express the model parameters as time-dependent functions. Using this approach, it is possible to predict the distributions of axon area and myelin sphericity for time points with no experimental image data available. This is especially useful as acquisition of image data for multiple time points can be time-consuming and expensive.

We showed that the distributions of axon area and myelin sphericity can be nicely approximated by (mixed) gamma and beta distributions, respectively. By assuming these models, the actual distribution of each, axon area and myelin sphericity, can be characterised by a small number of parameters per age, denoted by $\alpha_{1}, \alpha_{2}, \beta_{1}, \beta_{2}, \omega$ and $a, b$. For the joint distribution of both properties, a further parameter $\theta$ is considered, based on the copula approach discussed earlier. In Fig. 7, the values of these parameters are shown as blue dots for the ages of 6,12 and 24 months. Assuming that the same type of parametric models would describe the distributions of axon area and myelin sphericity for non-measured ages as well, we can interpolate the parameter values to obtain estimates of them for not observed ages. Fig. 7 shows the interpolated functions based on 4 different interpolation techniques.

The four candidates for an interpolation technique comprise of a piecewise linear interpolation, a general spline interpolation, a modified Akima interpolation (makima, Akima (1974)) and a piecewise cubic hermite interpolating polynomial (pchip, Carlson and Fritsch (1985)). As we have only 3 observed values, choosing a proper interpolation technique is challenging. Based on visual inspection of the emergent characteristics, i.e., mean and standard deviation, we can assess plausibility of the different techniques and give a range of possible values.

In Fig. 7, the calculated means and standard deviations of axon area and sphericity based on these interpolations are shown. Especially for the mean sphericity, pchip seems to yield unreasonable results and thus should not be used for interpolation. To a lesser extent, the same holds for linear interpolation. Thus, an interpolation technique similar to spline or makima seems to be best suited to the problem at hand. As makima reduces undulations in the interpolated curve and no data is available to support the excessive undulations introduced by regular spline interpolation, we choose the akima technique for interpolation. 

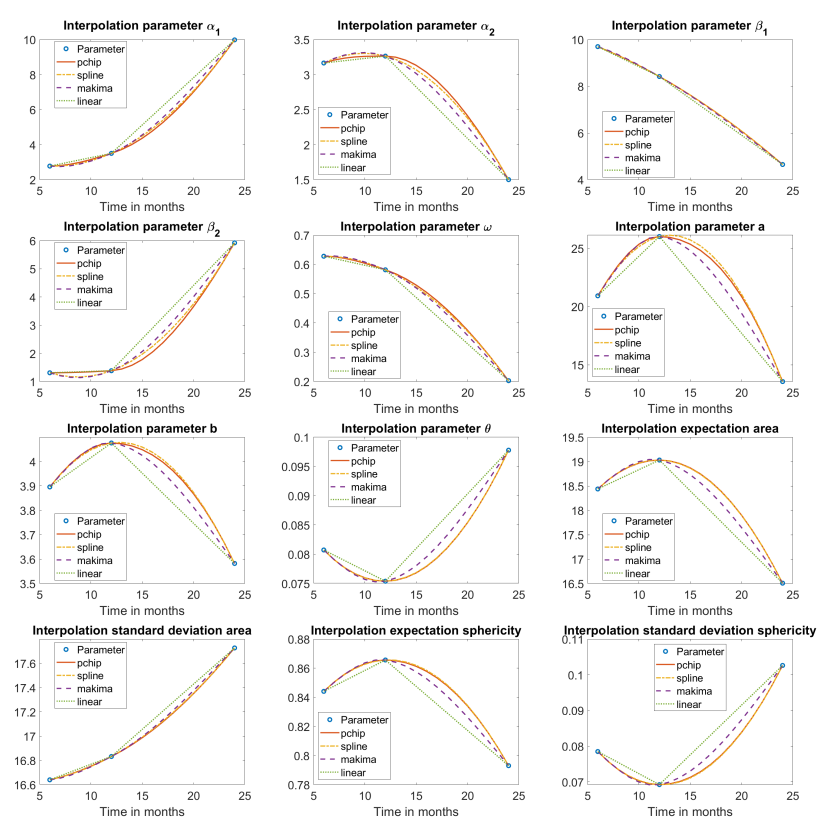

Fig. 7. Time-dependent functions for the model parameters $\alpha_{1}, \alpha_{2}, \beta_{1}, \beta_{2}, \omega, a, b, \theta$, using different interpolation techniques and resulting expectations and standard deviations for area and sphericity. Blue dots indicate values estimated from data, used as support points for the interpolation.

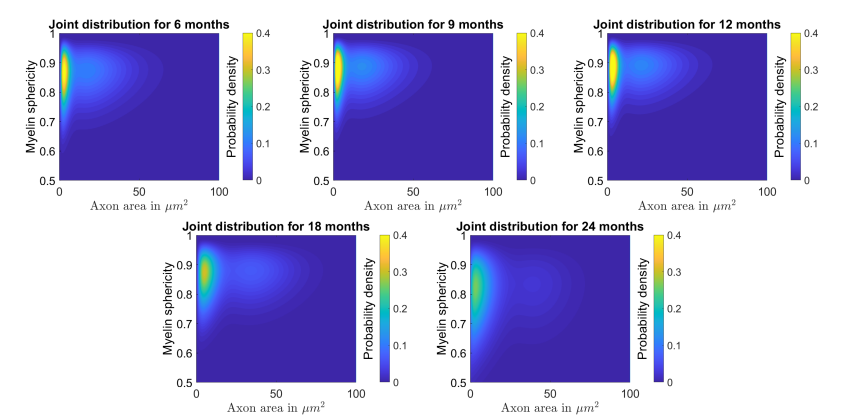

Fig. 8. Comparison of parametric joint distributions for axon area and sphericity of 6, 9, 12, 18 and 24 months old nerves. For 6, 12 and 24 months old nerves, these are based on the fitted parametric marginal (mixed) gamma and beta distributions and Clayton copulas.

Using the interpolated time-dependent parameter functions visualised in Fig. 7 based on makima interpolation, we can predict the joint distribution of axon area and myelin sphericity for ages not observed in experimental image data. Fig. 8 shows the predicted distributions for 9 and 18 months old nerves along with the fitted distributions for 6,12 , and 24 months. The corresponding mean values of axon area and myelin sphericity are given in Table 5 .

\begin{tabular}{|c||c|c|c|c|c|}
\hline age & 6 & 9 & 12 & 18 & 24 \\
\hline area & 18.47 & 17.86 & 19.05 & 22.19 & 16.51 \\
\hline sphericity & 0.84 & 0.86 & 0.86 & 0.85 & 0.79 \\
\hline
\end{tabular}

Table 5. Mean values of axon area and myelin sphericity for 6, 9, 12, 18 and 24 months old nerves. Note that, while being calculated from the copula model, the values for 6, 12, and 24 months seem to match the empirical mean values from Table 1.

\section{DISCUSSION}

Segmentation of images of granular structures composed of highly non-spherical objects poses serious challenges, especially when image resolution, contrast and artefacts are taken into account. In the present paper, we developed an automated segmentation algorithm for these types of structures which made use of a specifically adapted watershed method. The proposed method requires only little manual processing. We successfully applied this technique to images of cross-sections of femoral quadriceps nerves of mice to extract morphological properties of axons and the subcellular structures surrounding axons, so-called myelin sheaths, which play an important role in the propagation of action potentials. Here, our method proved robust against potential artefacts in the data. We extracted size and shape information of more than 5100 axons and myelin sheaths from image data of 6,12 and 24 months old mice. From this information, we determined two relevant geometrical descriptors, axon area and sphericity of the myelin sheaths. A subsequent statistical analysis of these datasets showed a clear change of axon size and myelin sphericity due to ageing. This confirms the expectation that ageing leads to degradation of axons into clover-like shape. Furthermore, we fitted parametric distribution models to the histograms of axon area and myelin sphericity and described the joint bivariate distribution of these geometrical descriptors using copulas. We found that the distribution of each, axon area and myelin sphericity, as well as the corresponding copula can be approximated by the same model type for all ages. This made it possible to describe the joint bivariate distribution for each age by eight parameters. Using this parametric representation of the data, we were able to further investigate the ageing-induced changes in size and shape. In particular, we found that that small axons tend to have less round myelin sheaths than medium and large axons and that this effect gets stronger for later ages. Finally, we proposed a method of parameter interpolation to predict the distribution of geometrical nerve descriptors for ages where no experimental image data is available. 


\section{ACKNOWLEDGEMENTS}

We are grateful to Drs. Dennis Klein, Xidi Yuan and Rudolf Martini (University Hospital of Würzburg) for providing low power multiple image alignments (MIA) of electron micrographs of nerves from mice of different ages, and for helpful comments on an earlier version of the manuscript. We thank the reviewers for their helpful comments on improving the manuscript.

\section{REFERENCES}

Akima H (1974). A method of bivariate interpolation and smooth surface fitting based on local procedures. Commun ACM 17:18-20.

Beucher S, Lantuéjoul C (1979). Use of watersheds in contour detection. In: Proceedings of the International Workshop on Image Processing, Real-Time Edge and Motion Detection/Estimation. Rennes, France.

Bickel PJ, Doksum KA (2015). Mathematical Statistics: Basic Ideas and Selected Topics, vol. 1. CRC Press, 2nd ed.

Carlson RE, Fritsch FN (1985). Monotone piecewise bicubic interpolation. SIAM J Numer Anal 22:386-400.

Durante F, Sempi C (2010). Copula therory: An introduction. In: Jaworski P, Durante F, Härdle W, Rychlik T, eds., Copula Theory and Its Application. Springer, 3-31.

Friede R (1986). Relation between myelin sheath thickness, internode geometry, and sheath resistance. Exp Neurol 92:234-47.

Furat O, Leißner T, Bachmann K, Gutzmer J, Peuker U, Schmidt V (2019a). Stochastic modeling of multidimensional particle characteristics using parametric copulas. Microsc Microanal 25:72034.

Furat O, Prifling B, Westhoff D, Weber M, Schmidt V (2019b). Statistical 3D analysis and modeling of complex particle systems based on tomographic image data. Prakt Metallogr Pr M 56:787-96.

Gonzalez RC, Woods RE (2007). Digital Image Processing. Pearson.

Heumans H, Nacken P, Toet A, Vincent L (1992). Graph morphology. J Vis Commun Image R 3:2438.

Hoshen J, Kopelman R (1976). Percolation and cluster distribution. i. cluster multiple labeling technique and criticalconcentration algorithm. Phys Rev $14: 3438-45$.
Joe H (1997). Multivariate Models and Multivariate Dependence Concepts. Chapman \& Hall.

Lantuéjoul C (1982). Geodesic segmentation. In: Uhr L, Preston K, eds., Multicomputers and Image Processing: Algorithms and Programs. Academic Press, 111-24.

Neumann M, Machado-Charry E, Zojer K, Schmidt V (2021). On variability and interdependence of local porosity and local tortuosity in porous materials: a case study for sack paper. Methodol Comput Appl 23:613-27.

Roerdink JB, Meijster A (2000). The watershed transform: Definitions, algorithms and parallelization strategies. Fund Inform 41:187228.

Sklar A (1959). Fonctions de répartition à $n$ dimensions et leurs marges. Publications de IInstitut Statistique de 1Université de Paris 8:22931.

Smith K, Blakemore W, Murray J, Patterson R (1982). Internodal myelin volume and axon surface area: A relationship determining myelin thickness? J Neurol Sci 55:231-46.

Soille P (2013). Morphological Image Analysis: Principles and Applications. Springer.

Vincent L (1989). Graphs and mathematical morphology. Signal Process 16:365-88.

von Loeper F, Schaumann P, de Langlard M, Hess R, Bäsmann R, Schmidt V (2020). Probabilistic prediction of solar power supply to distribution networks, using forecasts of global horizontal irradiation. Sol Energy 203:145-56.

Wadell H (1932). Volume, shape, and roundness of rock particles. J Geol 40:443-51.

Weber M, Bäuerle A, Schmidt M, Neumann M, Fändrich M, Ropinski T, Schmidt V (2020). Automatic identification of crossovers in cryo-EM images of murine amyloid protein A fibrils with machine learning. J Microsc Oxford 277:12-22.

Yuan X, Klein D, Kerscher S, West B, J. Weis IK, Martini R (2018). Macrophage depletion ameliorates peripheral neuropathy in aging mice. J Neurosci 38:4610-20.

Zaimi A, Wabartha M, Herman V, Antonsanti PL, Perone CS, Cohen-Adad J (2018). AxonDeepSeg: automatic axon and myelin segmentation from microscopy data using convolutional neural networks. Sci Rep UK 8:1-11. 